\title{
Principles of the human body functioning and their applications in integrative medicine (review)
}

\begin{abstract}
A new developing branch of medicine - Integrative Medicine - gains popularity in the world. Thousands of doctors are using this approach in their practice helping people to be healthy till very long age. Hundreds of papers are being published discussing different methods and technologies. In this paper we present review of the overall principles of body functioning related to Integrative Medicine, which allows to understand the scope of applications of different treatment modalities.
\end{abstract}

Volume 5 Issue 6 - 2018

\author{
Korotkov KG \\ St. Petersburg Federal University of Information Technologies, \\ Mechanics and Optics, Russia \\ Correspondence: Konstantin G Korotkov, St. Petersburg \\ Research Institute of Physical Culture and Sport, NIIFK, Ligovski \\ prospect 56E, St. Petersburg, 19104, Russia, Tel +7-9219368394, \\ Fax+7-8I26004II7, Email koratkov2000@gmail.com
}

Received: July 28, 2018| Published: November 27, 2018

\section{Introduction}

Throughout the history of modern science, scientists have tried to understand the principles that govern the operation of the human body. They have always done so on the basis of existing concepts, such as the current accepted ruling paradigm. In the XVII - XIXth centuries, the human body was compared with mechanisms, functioning on the basis of Newtonian principles. In the XXth century, biochemical principles came to the forefront, supported to some extent by the electrical; in the XXIst century, the temptation is to reduce all the roots of health problems to genetics, but this approach has not been justified. At last, we have come to the understanding that all these principles operate simultaneously, and all of them are equally important, and influence each other. Chemical agents circulate in the body, controlled by electrical signals, while factoring the effects of genetics and local environmental conditions. These principles have been accepted by a new developing branch of medicine - Integrative Medicine. It is defined by the Consortium of Academic Health Centers for Integrative Medicine as "an approach to the practice of medicine that makes use of the best-available evidence taking into account the whole person (body, mind and spirit), including all aspects of lifestyle. ${ }^{1}$

Concepts of scientific integrative medicine include ${ }^{2}$ (i) negative feedback regulation, maintaining stability of the body's monitored variables; (ii) homeostats, which compare information about monitored variables with algorithms for responding; (iii) multiple effectors, enabling compensatory activation of alternative effectors and primitive specificity of stress response patterns; (iv) effector sharing, accounting for interactions among homeostats and phenomena such as hyperglycemia attending gastrointestinal bleeding and hyponatremia attending congestive heart failure; (v) stress, applying a definition as a state rather than as an environmental stimulus or stereotyped response; (vi) distress, using a noncircular definition that does not presume pathology; (vii) allostasis, corresponding to adaptive plasticity of feedback-regulated systems; and (viii) allostatic load, explaining chronic degenerative diseases in terms of effects of cumulative wear and tear.

Detailed discussion of the methods and therapies utilized in Integrative Medicine may be found $\mathrm{in}^{3}$ here I want to discuss the overall principles of body functioning related to Integrative Medicine. The body is an open system, constantly exchanging matter, energy and information with the environment, ranging from individuals, like ourselves, to the level of the universe; dependent on the absorbed water, air, and food; and, as has now been established, on the wide range of unseen fields of natural and man-made origin. One cannot isolate people from their environment. Similarly, the workings of the kidneys or the stomach cannot be considered without taking into account the activity of the organism as a whole. At the same time, we have not yet addressed the influence of consciousness and soul upon the physical body and won't for a while as scientific studies on this matter have only recently begun.

Speaking of the body from a conceptual point of view, we can simplistically distinguish three main operational levels: external material and information factors, the control loop, and the level of executive systems. The following can be attributed to the external material and information factors: ${ }^{4}$

a. The information field of the universe;

b. The information field of human consciousness;

c. Informational influence of society and people on each other;

d. Individual human responses, emotions and stress;

e. Effects of food and water;

f. The impact of natural and technological fields;

g. The level of trace elements accumulated in the body;

h. Chemical and radiation pollution;

i. Viruses, bacteria, parasites, worms, fungi, mold and other living entities that cause trouble;

j. Influence of geophysical conditions of the environment and habitats.Marked effects are processed by the loop of response and management, which consists of three main systems:

k. The nervous system (the brain as part of the nervous system);

1. The endocrine system; and

m. The immune system.

These systems control the entire human physiology. They respond to all the attacks coming from the external environment and react to changes in external conditions, struggling to cope with the food consumed while reflecting our emotional and stress responses. And so it goes on 24 hours a day, seven days a week, for years on end. We know that the body can work for $100+$ years and the number of people who have reached this level in the world is constantly growing. There are talks that this period may be extended to 130-140 years, but so far it has remained in the domain of wishful thinking. And also, 
there is the question whether it is advisable to live so long. You will be tired after all... The main thing is to stay active in your old age and do not lie down for decades as a vegetable.

As with any control system, the distinguished triad (nervousendocrine-immune) accumulates errors that affect normal operations - that is, the state of health. This mainly occurs when a person is under the influence of harmful factors over a period of many years. Harmful factors include consistently consuming fast food products and chemicals, often working on the night shift or living in a state of permanent stress. The body resists for a long time, but eventually succumbs. Recovery is possible, but it requires much time and great effort.

Sometimes, for unknown reasons, the system malfunctions. The immune system begins to fight with its own cells, and there is a complex class of autoimmune diseases. In some cases, it depends on the genetic predetermination, but mostly it remains a mystery to modern medicine. It can only register the fact of damage, and then attempt to block the process with strong chemicals. Decoding the genome was the greatest achievement of science, but the assurance that this would allow us to overcome all diseases has not been justified. Attempts to turn genetics into a business and register patents on individual genes, have, fortunately, failed. Everything is much more difficult than was envisaged by linear-minded biologists. There is no aging gene or gene of genius. The situation is much more complex. (Though there are a number of genes that define predisposition to certain diseases, for example, breast cancer). In short, instead of a cavalry charge, it has been necessary to organize a slow attack.

Much attention is paid now to the direction called epigenetics ${ }^{5-7}$ the process of the expression of genes influenced by external factors. That is important not only for the genes which are in the genome but whether they will be turned on at some stage in life. This largely depends on the influence of the environment. If once revealed, a new feature may be transferred by inheritance. Science, at this exciting new stage, has come to recognize the ideas of Jean- Baptiste Lamarck (1744-1829) that accumulated traits may be inherited. This idea had been completely driven under-ground by the pressure of Darwinism. Lamarck was mocked and forgotten (although his monument stands at the center of Paris). Only now we are coming back to his ideas.All these three control systems are interconnected, continuously interact, exchange information and influence each other. This is a united control system of the body. Under its control are the executive systems; the main ones are: ${ }^{8}$

a. The cardiovascular system;

b. Gastro-intestinal tract;

c. Respiratory system;

d. Circulatory system;

e. Muscular-skeletal system;

f. Excretory system;

\section{g. Skin system.}

This division is very conditional. In the literature can be found different classifications, but these do not matter to us. What is the most important is the idea of the functioning circuits. Our body is a unified system in which everything is interconnected and interdependent. Disturbances in one system instantaneously affect the workings of all the others and only together can we try to fix the situation. We can say that the basic idea of the body's functioning is the maintenance of homeostasis: the balance of all systems with the environment. Our body possesses huge compensatory abilities and, in the case of the loss of any function, can eventually be replaced by others. For example, the loss of one kidney or one lung is compensated by the work of the remaining organ. Recently, it has been shown that even nerve cells could regenerate! ${ }^{9,10}$ Related to the brain, this concept is known as the brain plasticity, ${ }^{11}$ the ability of different parts of the brain to replace damaged parts. Therefore, with appropriate therapy, a person could recover from a stroke fairly completely.

Another vision of the functioning of the human body is the Oriental approach, which operates on the concept of energy and describes energy's movement in the human body. In Traditional Chinese medicine, this corresponds to the principle of Yin-Yang, which has a correlation with the activity of the sympathetic and parasympathetic nervous systems. The energy is transferred through the meridians, which are divided into Yin and Yang. Active points of meridians are acupuncture points through which impacts can be made via the meridian on a particular organ. In the twentieth century, it was found that the resistance of the skin at these points is significantly less than the surrounding regions. This fact was completely inexplicable from the standpoint of Occidental science and caused Western scientists and physicians take Chinese medicine more seriously. The scientific world was divided into those who have accepted the principles of the movement of energy in the body and are using them, and those who deny these categorically. In Indian Ayurvedic medicine, there is the principle of Chakras - the energy centers that control all the processes in the body - which is very similar to the principle of vegetative regulation. At the same time, we need to keep in mind the levels of human body functions and the hierarchy of their response to the external intrusions (Figure 1). ${ }^{12}$

The first is on a quantum level: electrons and photons exchange in the body..$^{13}$ This is the most sensitive level and responds both to the material and informational influences. Deregulation at this level may cause an inability of mitochondria to generate energy, and people will consequently suffer from low energy, having all classical parameters in the normal range (typical for chronic fatigue). Treatment of problems at this level should be done using devices and methods of Quantum Medicine. ${ }^{14}$ These are first of all different devices using all spectrum of light - from UV to infrared, both non-coherent and laser. The biophysical principles of light interaction with biological tissues are the topic of intensive scientific studies. ${ }^{15}$ Next group of quantum devices are apparatus using low intensity electromagnetic waves of different frequencies. Between them one of the most efficient line of devices using Extremely High Frequencies (EHF) in the Gigahertz range. EHF therapy studies the mechanisms of action on living organism of electromagnetic radiation (EMR) of millimetric type (1$10 \mathrm{~mm}$ ) at high-frequency $(30-300 \mathrm{GHz})$ of low intensity, and medical practice that uses the effects of these electromagnetic waves as a treatment of various disorders. ${ }^{16}$

The next level is the level of the Energy field (Biofield). ${ }^{17}$ The term originated out of a group convened by the National Institutes of Health (NIH) Office of Alternative Medicine in the early 1990s in an attempt to provide an organizing schema for a wide range of healing practices, often referred to as energy medicine or bioenergetic therapies ${ }^{18}$ and is widely used nowadays in Integrative Medicine. This level reflects participation of different dimensions of a person functioning: physical, mental and spiritual. Devices using electromagnetic, magnetic, acoustic fields, as well as light and music, are effective at this level. A combination of different types of treatments have the most beneficial effect, as an organism may select the optimal influence. Specific methods should be used to influence mental and spiritual levels of a person, and the response of the Energy field would indicate the effect of the treatment. At the same time therapies such as botanicals, supplements, mind-body, lifestyle choices, nutrition, exercise, spirituality are widely used as well. ${ }^{19}$ Electrophotonic Analysis is the optimal method of study at this level. ${ }^{20,21}$ 
At the Chemical level we see a deregulation of different molecular agents operating in the body: from neurotransmitters to catecholamines, hormones, etc. This is very important as all our functioning levels depend on their production. Modern medicine has many instruments to detect problems here and all allopathic medicine is designed to influence the chemical processes directly. In many cases, without the involvement of the previous levels, the modern treatment does not allow to remove the cause of the problem and, hence, can transform the illness into a chronic situation. When all the previous levels are deregulated for a long time, symptoms appear. As we see, this is the last stage of a long-lasting drama and, at this point, people often begin feeling the effects. This is usually when people eventually reach out to doctors. However, if we want to have an effective treatment process, we need to address all the levels simultaneously. In short, the more differential the combination of all the available methods of treatment are used, the better the results.

\section{Conclusion}

In the XXth century medicine achieved tremendous results: a lot of grave deceases was successfully treated and practically vanished in civilized countries. Heart and liver transplants became everyday operations. We can write the long list of great achievements. At the same time new problems emerged and with them new understanding of the human health and well-being. Integrative Medicine approach should serve as one of the branches of modern medicine, helping to solve the problem of developing healthy and happy population.

\section{Acknowledgments}

None.

\section{Conflicts of interest}

The author declares there are no conflicts of interest.

\section{References}

1. White JD. Complementary and Alternative Medicine. Abeloff's Clinical Oncology. $5 \mathrm{t}^{\mathrm{h}}$ ed. Churchill-Livingstone, London; 2014. p. 519-529.

2. Goldstein DS. Concepts of Scientific Integrative Medicine Applied to the Physiology and Pathophysiology of Catecholamine Systems. Compr Physiol. 2013;3(4):1569-1610.

3. Rakel D. Integrative Medicine. Elsevier, NY; 2018.

4. Korotkov KG. The Energy of Consciousness. Amazon.com Publishing; 2012.
5. DupontC,ArmantDR, BrennerCA. Epigenetics: definition, mechanisms and clinical perspective. Semin Reprod Med. 2009;27(5):351-357.

6. Reik W. Stability and flexibility of epigenetic gene regulation in mammalian development. Nature. 2007;447(7143):425-432.

7. Bishop KS, Huawen Xu, Marlow G. Epigenetic Regulation of Gene Expression Induced by Butyrate in Colorectal Cancer: Involvement of MicroRNA. Epigenetic Insights. 2017.

8. Hall JE. Guyton and Hall Textbook of Medical Physiology (Guyton Physiology). 13th ed. Elsevier, NY; 2016.

9. Steward MM, Sridhar A, Mejer JS. Neural Regeneration. Curr Top Microbio Immunol. 2013;367:163-191.

10. Recknor JB, Mallapragada SK. Nerve Regeneration: Tissue Engineering Strategies. In: Bronzino JD, editor. The biomedical engineering handbook. 3rd ed. Boca Raton, Fla CRC Taylor \& Francis; 2006.

11. Sasmita AO, Kuruvilla J, Ling AP. Harnessing neuroplasticity: modern approaches and clinical future. The International Journal of Neuroscience: 2018;4:1-17.

12. Korotkov K. The Energy of Health. Amazon.com Publishing;. 2017.

13. Korotkov K, Williams B, Wisneski L. Biophysical Energy Transfer Mechanisms in Living Systems: The Basis of Life Processes. $J$ of Alternative and Complementary Medicine. 2004;10 (1):49-57.

14. Wisneski LA, Anderson L. The Scientific Basis of Integrative Medicine. 4th ed. CRC Press; 2017.

15. Smith K. The Science of Photobiology. Springer Science \& Business Media; 2013.

16. Teppone M, Avakian R, Novikova L, Grigoriev S. Extremely High Frequency (EHF) Therapy. The Alt Compl J. 1963;16(11): 29-35.

17. Rubik B. The Biofield: Bridge Between Mind And Body. The J of Natural and Social Philosophy. 2015;11(2):83-96.

18. Kreitzer MJ, Saper R. Exploring the Biofield. Global Adv Health Med. 2015;(4):3-4.

19. Galitzer M, Trivieri L. Outstanding Health. AHI Publishing; 2015.

20. Jakovleva E, Korotkov K, Electrophotonic Analysis in Medicine. GDV Bioelectrography research. Amazon.com Publishing; 2012.

21. Korotkov KG. Energy fields Electrophotonic analysis in humans and nature. Amazon com Publishing; 2012. 